\title{
Acoustic Spectroscopy, Acoustic Resonance Spectroscopy and Auger Spectroscopy Comparative Study on Anti-Cancer Nano Drugs Delivery in Malignant and Benign Human Cancer Cells and Tissues with the Passage of Time under Synchrotron Radiation
}

\author{
Alireza Heidari ${ }^{1^{*}}$ \\ Faculty of Chemistry, California South University, 14731 Comet St. Irvine, CA 92604, USA, E-mail: Alireza.Heidari@calsu.us, Scholar. \\ Researcher.Scientist@gmail.com
}

Received: 29 November, 2017; Accepted: 19 April, 2018; Published: 25 April, 2018

*Corresponding author: Alireza Heidari, Faculty of Chemistry, California South University, 14731 Comet St. Irvine, CA 92604, USA, E-mail: Scholar. Researcher.Scientist@gmail.com,Alireza.Heidari@calsu.us

\begin{abstract}
In the current study, we have experimentally and comparatively investigated and compared anti-cancer Nano drugs delivery in malignant human cancer cells and tissues before and after irradiating of synchrotron radiation using Acoustic Spectroscopy, Acoustic Resonance Spectroscopy and Auger Spectroscopy.
\end{abstract}

\section{Introduction}

In the current study, we have experimentally and comparatively investigated and compared anti-cancer Nano drugs delivery in malignant human cancer cells and tissues before and after irradiating of synchrotron radiation using Acoustic Spectroscopy, Acoustic Resonance Spectroscopy and Auger Spectroscopy. It is clear that malignant human cancer cells and tissues have gradually transformed to benign human cancer cells and tissues under synchrotron radiation with the passage of time (Figures 1-3) [1-150].

\section{Results and Discussion}

It should be noted that malignant human cancer cells and tissues were exposed under white synchrotron radiation for 30 days. Furthermore, there is a shift of the spectrum in all of spectra after irradiating of synchrotron radiation that it is because of the malignant human cancer cells and tissues shrink post white synchrotron irradiation with the passage of time. In addition, all of the figures are related to the same human cancer cells and tissues. Moreover, in all of the figures $y$-axis shows intensity and also $\mathrm{x}$-axis shows energy (keV).

\section{Conclusion}

It can be concluded that malignant human cancer cells and tissues have gradually transformed to benign human cancer cells and tissues under synchrotron radiation with the passage of time (Figures 1-3).

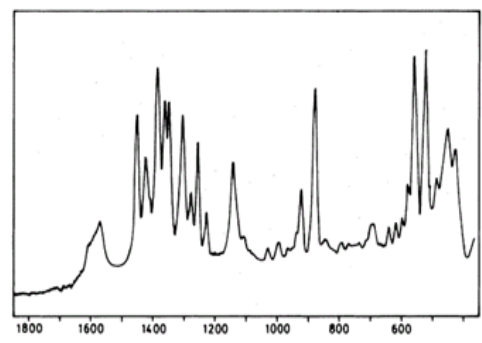

(a)

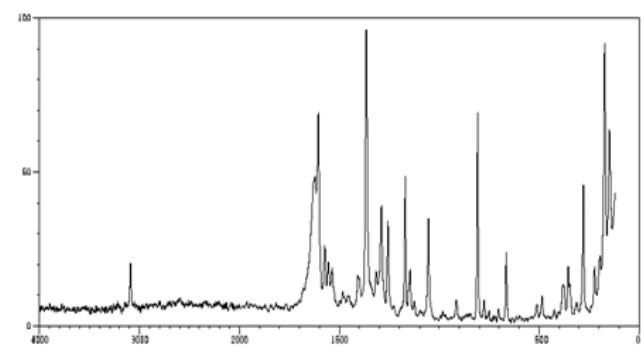

(b)

Figure 1: Acoustic Spectroscopy analysis of malignant human cancer cells and tissues

(a) before and (b) after irradiating of synchrotron radiation in transformation process to benign human cancer cells and tissues with the passage of time [1-150]. 


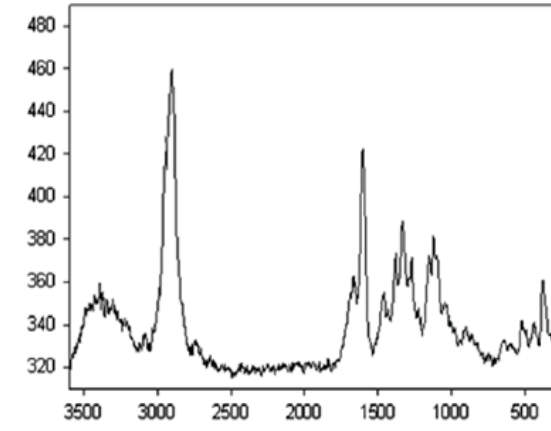

(a)

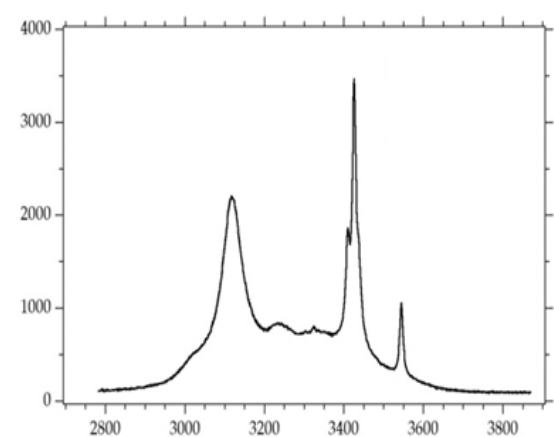

(b)

Figure 2: Acoustic Resonance Spectroscopy analysis of malignant human cancer cells and tissues

(a) before and (b) after irradiating of synchrotron radiation in transformation process to benign human cancer cells and tissues with the passage of time [1-150].

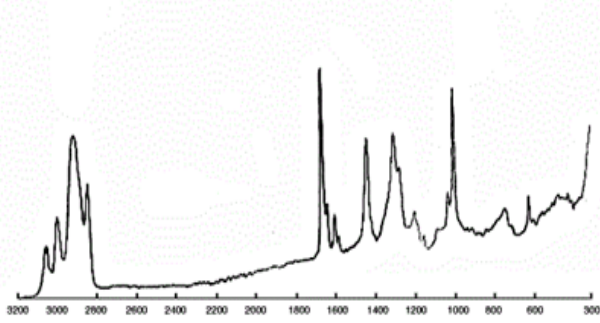

(a)

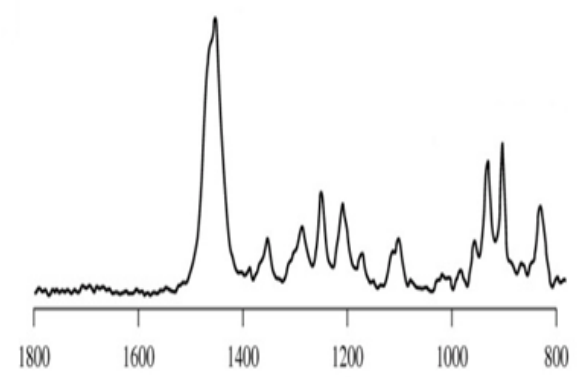

(b)

Figure 3: Auger Spectroscopy analysis of malignant human cancer cells and tissues

(a) before and (b) after irradiating of synchrotron radiation in transformation process to benign human cancer cells and tissues with the passage of time [1-150].

\section{References}

1. Heidari A, Brown C. Study of Composition and Morphology of Cadmium Oxide (CdO) Nanoparticles for Eliminating Cancer Cells. J Nanomed Res. 2015;2(5):1-20.

2. A Heidari, C Brown. Study of Surface Morphological, Phytochemical and Structural Characteristics of Rhodium (III) Oxide (Rh203) Nanoparticles. International Journal of Pharmacology, Phytochemistry and Ethnomedicine. 2015;1:15-19. doi:10.18052/ www.scipress.com/IJPPE.1.15

3. Heidari A. An Experimental Biospectroscopic Study on Seminal Plasma in Determination of Semen Quality for Evaluation of Male Infertility. Int J Adv Technol. 2016;7:e007. doi: 10.4172/0976$4860.1000 \mathrm{e} 007$

4. Heidari A. Extraction and Preconcentration of N-Tolyl-SulfonylPhosphoramid-Saeure-Dichlorid as an Anti-Cancer Drug from Plants: A Pharmacognosy Study. J Pharmacogn Nat Prod. 2016;2:e103. doi: 10.4172/2472-0992.1000e103

5. Heidari A. Thermodynamic Study on Hydration and Dehydration of DNA and RNA-Amphiphile Complexes. J Bioeng Biomed Sci S. 2016;006. doi:10.4172/2155-9538.S3-006

6. Heidari A. Computational Studies on Molecular Structures and Carbonyl and Ketene Groups' Effects of Singlet and Triplet Energies of Azidoketene $\mathrm{O}=\mathrm{C}=\mathrm{CHNNN}$ and Isocyanatoketene $\mathrm{O}=\mathrm{C}=\mathrm{CHN}=\mathrm{C}=\mathrm{O}$. J Appl Computat Math. 2016;5:e142. doi:10.4172/21689679.1000e 142

7. Heidari A. Study of Irradiations to Enhance the Induces the Dissociation of Hydrogen Bonds between Peptide Chains and Transition from Helix Structure to Random Coil Structure Using ATR-FTIR, Raman and 1HNMR Spectroscopies. J Biomol Res Ther. 2016;5(2):1-3. doi:10.4172/2167-7956.1000e146

8. Heidari A. Future Prospects of Point Fluorescence Spectroscopy, Fluorescence Imaging and Fluorescence Endoscopy in Photodynamic Therapy (PDT) for Cancer Cells. J Bioanal Biomed. 2016;8:e135. doi: 10.4172/1948-593X.1000e135

9. Heidari A. A Bio-Spectroscopic Study of DNA Density and Color Role as Determining Factor for Absorbed Irradiation in Cancer Cells. Adv Cancer Prev. 2016;1:e102. doi:10.4172/acp.1000e102

10. Heidari A. Manufacturing Process of Solar Cells Using Cadmium Oxide (CdO) and Rhodium (III) Oxide (Rh203) Nanoparticles. J Biotechnol Biomater. 2016;6:e125. doi:10.4172/2155-952X.1000e125

11. Heidari A. A Novel Experimental and Computational Approach to Photobiosimulation of Telomeric DNA/RNA: A Biospectroscopic and Photobiological Study. J Res Development. 2016;4:144. doi: 10.4172/2311-3278.1000144 
12. Heidari A. Biochemical and Pharmacodynamical Study of Microporous Molecularly Imprinted Polymer Selective for Vancomycin, Teicoplanin, Oritavancin, Telavancin and Dalbavancin Binding. Biochem Physiol. 2016;5:e146. doi: 10.4172/21689652.1000e146

13. Heidari A. Anti-Cancer Effect of UV Irradiation at Presence of Cadmium Oxide (CdO) Nanoparticles on DNA of Cancer Cells: A Photodynamic Therapy Study. Arch Cancer Res. 2016;4:1.

14. Heidari A. Biospectroscopic Study on Multi-Component Reactions (MCRs) in Two A-Type and B-Type Conformations of Nucleic Acids to Determine Ligand Binding Modes, Binding Constant and Stability of Nucleic Acids in Cadmium Oxide (CdO) Nanoparticles-Nucleic Acids Complexes as Anti-Cancer Drugs. Arch Cancer Res. 2016;4:2.

15. Heidari A. Simulation of Temperature Distribution of DNA/RNA of Human Cancer Cells Using Time- Dependent Bio-Heat Equation and Nd: YAG Lasers. Arch Cancer Res. 2016;4:2.

16. Heidari A. Quantitative Structure-Activity Relationship (QSAR) Approximation for Cadmium Oxide (CdO) and Rhodium (III) Oxide (Rh203) Nanoparticles as Anti-Cancer Drugs for the Catalytic Formation of Proviral DNA from Viral RNA Using Multiple Linear and Non-Linear Correlation Approach. Ann Clin Lab Res. 2016;4:1.

17. Heidari A. Biomedical Study of Cancer Cells DNA Therapy Using Laser Irradiations at Presence of Intelligent Nanoparticles. J Biomedical Sci. 2016;5:2. doi:10.4172/2254-609X.100023

18. Heidari A. Measurement the Amount of Vitamin D2 (Ergocalciferol), Vitamin D3 (Cholecalciferol) and Absorbable Calcium (Ca2+), Iron (II) $(\mathrm{Fe} 2+)$, Magnesium (Mg2+), Phosphate (PO4-) and Zinc (Zn2+) in Apricot Using High-Performance Liquid Chromatography (HPLC) and Spectroscopic Techniques. J Biom Biostat. 2016;7:292. doi:10.4172/2155-6180.1000292

19. Heidari A. Spectroscopy and Quantum Mechanics of the Helium Dimer $(\mathrm{He} 2+)$, Neon Dimer (Ne2+), Argon Dimer (Ar2+), Krypton Dimer (Kr2+), Xenon Dimer (Xe2+), Radon Dimer(Rn2+) and Ununoctium Dimer (Uuo2+) Molecular Cations. Chem Sci J. 2016;7:e112. doi:10.4172/2150-3494.1000e112

20. Heidari A. Human Toxicity Photodynamic Therapy Studies on DNA/ RNA Complexes as a Promising New Sensitizer for the Treatment of Malignant Tumors Using Bio-Spectroscopic Techniques. J Drug Metab Toxicol. 2016;7:e129. doi:10.4172/2157-7609.1000e129

21. Heidari A. Novel and Stable Modifications of Intelligent Cadmium Oxide (CdO) Nanoparticles as Anti- Cancer Drug in Formation of Nucleic Acids Complexes for Human Cancer Cells Treatment. Biochem Pharmacol (Los Angel). 2016;5:207. doi:10.4172/21670501.1000207

22. Heidari A. A Combined Computational and QM/MM Molecular Dynamics Study on Boron Nitride Nanotubes (BNNTs), Amorphous Boron Nitride Nanotubes (a-BNNTs) and Hexagonal Boron Nitride Nanotubes (h-BNNTs) as Hydrogen Storage. Struct Chem Crystallogr Commun. 2016;2:1.

23. Heidari A. Pharmaceutical and Analytical Chemistry Study of Cadmium Oxide (CdO) Nanoparticles Synthesis Methods and Properties as Anti-Cancer Drug and its Effect on Human Cancer Cells. Pharm Anal Chem Open Access. 2016;2:113. doi: 10.4172/24712698.1000113

24. Heidari A. A Chemotherapeutic and Biospectroscopic Investigation of the Interaction of Double- Standard DNA/RNA-Binding Molecules with Cadmium Oxide (CdO) and Rhodium (III) Oxide (Rh203) Nanoparticles as Anti-Cancer Drugs for Cancer Cells' Treatment. Chemo Open Access. 2016;5: e129. doi:10.4172/2167$7700.1000 \mathrm{e} 129$

25. Heidari A. Pharmacokinetics and Experimental Therapeutic Study of DNA and Other Biomolecules Using Lasers: Advantages and Applications. J Pharmacokinet Exp Ther. 2016;1:e005. doi:10.4172/ jpet.1000e005

26. Heidari A. Determination of Ratio and Stability Constant of DNA/RNA in Human Cancer Cells and Cadmium Oxide (CdO) Nanoparticles Complexes Using Analytical Electrochemical and Spectroscopic Techniques. Insights Anal Electrochem. 2016;2:1.

27. Heidari A. Discriminate between Antibacterial and NonAntibacterial Drugs Artificial Neutral Networks of a Multilayer Perceptron (MLP) Type Using a Set of Topological Descriptors. J Heavy Met Toxicity Dis. 2016;1:2.

28. Heidari A. Combined Theoretical and Computational Study of the Belousov-Zhabotinsky Chaotic Reaction and Curtius Rearrangement for Synthesis of Mechlorethamine, Cisplatin, Streptozotocin, Cyclophosphamide, Melphalan, Busulphan and BCNU as Anti-Cancer Drugs. Insights Med Phys. 2016;1:2.

29. Heidari A. A Translational Biomedical Approach to Structural Arrangement of Amino Acids' Complexes: A Combined Theoretical and Computational Study. Transl Biomed. 2016;7:2.

30. Heidari. Ab Initio and Density Functional Theory (DFT) Studies of Dynamic NMR Shielding Tensors and Vibrational Frequencies of DNA/RNA and Cadmium Oxide (CdO) Nanoparticles Complexes in Human Cancer Cells. J Nanomedine Biotherapeutic Discov . 2016;6:e144. doi:10.4172/2155-983X.1000e144

31. Heidari A. Molecular Dynamics and Monte-Carlo Simulations for Replacement Sugars in Insulin Resistance, Obesity, LDL Cholesterol, Triglycerides, Metabolic Syndrome, Type 2 Diabetes and Cardiovascular Disease: A Glycobiological Study. J Glycobiol. 2016;5:e111. doi:10.4172/2168-958X.1000e111

32. Heidari A. Synthesis and Study of 5-[(Phenylsulfonyl)Amino]-1,3,4Thiadiazole-2-Sulfonamide as Potential Anti-Pertussis Drug Using Chromatography and Spectroscopy Techniques. Transl Med (Sunnyvale). 2016;6:e138. doi: 10.4172/2161-1025.1000e137

33. Heidari A. Nitrogen, Oxygen, Phosphorus and Sulphur Heterocyclic Anti-Cancer Nano Drugs Separation in the Supercritical Fluid of Ozone (03) Using Soave-Redlich-Kwong (SRK) and Pang-Robinson (PR) Equations. Electronic J Biol. 2016;12:3.

34. Heidari A. An Analytical and Computational Infrared Spectroscopic Review of Vibrational Modes in Nucleic Acids. Austin J Anal Pharm Chem. 2016;3(1):1058.

35. Heidari A, Brown C. Phase, Composition and Morphology Study and Analysis of Os-Pd/HfC Nanocomposites. Nano Res Appl. 2016;2:1.

36. Heidari A, Brown C. Vibrational Spectroscopic Study of Intensities and Shifts of Symmetric Vibration Modes of Ozone Diluted by Cumene. International Journal of Advanced Chemistry. 2016;4(1)59.

37. Heidari A. Study of the Role of Anti-Cancer Molecules with Different Sizes for Decreasing Corresponding Bulk Tumor Multiple Organs or Tissues. Arch Can Res. 2016;4:2. 
38. Heidari A. Genomics and Proteomics Studies of Zolpidem, Necopidem, Alpidem, Saripidem, Miroprofen, Zolimidine, Olprinone and Abafungin as Anti-Tumor, Peptide Antibiotics, Antiviral and Central Nervous System (CNS) Drugs. J Data Mining Genomics \& Proteomics. 2016;7: e125. doi:10.4172/2153-0602.1000e125

39. Heidari A. Pharmacogenomics and Pharmacoproteomics Studies of Phosphodiesterase-5 (PDE5) Inhibitors and Paclitaxel AlbuminStabilized Nanoparticles as Sandwiched Anti-Cancer Nano Drugs between Two DNA/RNA Molecules of Human Cancer Cells. J Pharmacogenomics Pharmacoproteomics. 2016;7:e153.

40. Heidari A. Biotranslational Medical and Biospectroscopic Studies of Cadmium Oxide (CdO) Nanoparticles-DNA/RNA Straight and Cycle Chain Complexes as Potent Anti-Viral, Anti-Tumor and AntiMicrobial Drugs: A Clinical Approach. Transl Biomed. 2016;7:2.

41. Heidari A. A Comparative Study on Simultaneous Determination and Separation of Adsorbed Cadmium Oxide (CdO) Nanoparticles on DNA/RNA of Human Cancer Cells Using Biospectroscopic Techniques and Dielectrophoresis (DEP) Method. Arch Can Res. 2016;4:2.

42. Heidari A. Cheminformatics and System Chemistry of Cisplatin Carboplatin, Nedaplatin, Oxaliplatin, Heptaplatin and Lobaplatin as Anti-Cancer Nano Drugs: A Combined Computational and Experimental Study. J Inform Data Min. 2016;1:3. doi: 10.21767/2472-1956.100015

43. Heidari A. Linear and Non-Linear Quantitative Structure-AntiCancer-Activity Relationship (QSACAR) Study of Hydrous Ruthenium (IV) Oxide (RuO2) Nanoparticles as Non-Nucleoside Reverse Transcriptase Inhibitors (NNRTIs) and Anti-Cancer Nano Drugs. J Integr Oncol. 2016;5:e110. doi:10.4172/2329-6771.1000e110

44. Heidari A. Synthesis, Characterization and Biospectroscopic Studies of Cadmium Oxide (CdO) Nanoparticles-Nucleic Acids Complexes Absence of Soluble Polymer as a Protective Agent Using Nucleic Acids Condensation and Solution Reduction Method. J Nanosci Curr Res. 2016;1(1):e101.

45. Heidari A. Coplanarity and Collinearity of 4'-Dinonyl-2,2'-Bithiazole in One Domain of Bleomycin and Pingyangmycin to be Responsible for Binding of Cadmium Oxide (CdO) Nanoparticles to DNA/RNA Bidentate Ligands as Anti-Tumor Nano Drug. Int J Drug Dev \& Res. 2016;8:007-008.

46. Heidari A. A Pharmacovigilance Study on Linear and Non-Linear Quantitative Structure (Chromatographic) Retention Relationships (QSRR) Models for the Prediction of Retention Time of AntiCancer Nano Drugs under Synchrotron Radiations. J Pharmacovigil. 2016;4:e161. doi:10.4172/2329-6887.1000e161

47. Heidari A. Nanotechnology in Preparation of Semipermeable Polymers. J Adv Chem Eng. 2016;6:157.

48. Heidari A. A Gastrointestinal Study on Linear and Non-Linear Quantitative Structure (Chromatographic) Retention Relationships (QSRR) Models for Analysis 5-Aminosalicylates Nano Particles as Digestive System Nano Drugs under Synchrotron Radiations. J Gastrointest Dig Syst. 2016;6:e119. doi:10.4172/2161069X.1000e119

49. Heidari A. DNA/RNA Fragmentation and Cytolysis in Human Cancer Cells Treated with Diphthamide Nano Particles Derivatives. Biomedical Data Mining. 2016;5:e102. doi:10.4172/20904924.1000e102
50. Heidari A. A Successful Strategy for the Prediction of Solubility in the Construction of Quantitative Structure-Activity Relationship (QSAR) and Quantitative Structure-Property Relationship (QSPR) under Synchrotron Radiations Using Genetic Function Approximation (GFA) Algorithm. J Mol Biol Biotechnol. 2016;1:1.

51. Alireza Heidari. Computational Study on Molecular Structures of C20, C60, C240, C540, C960, C2160 and C3840 Fullerene Nano Molecules under Synchrotron Radiations Using Fuzzy Logic. J Material Sci Eng. 2016;5(5):282.

52. Alireza Heidari. Graph Theoretical Analysis of Zigzag Polyhexamethylene Biguanide, Polyhexamethylene Adipamide, Polyhexamethylene Biguanide Gauze and Polyhexamethylene Biguanide Hydrochloride (PHMB) Boron Nitride Nanotubes (BNNTs), Amorphous Boron Nitride Nanotubes (a-BNNTs) and Hexagonal Boron Nitride Nanotubes (h-BNNTs). J Appl Computat Mat; 2016. doi:10.4172/2168-9679.1000e143

53. Alireza Heidari. The Impact of High Resolution Imaging on Diagnosis", Int J Clin Med Imaging ; 2016;3(6):1000e101.

54. Alireza Heidari. A Comparative Study of Conformational Behavior of Isotretinoin (13-Cis Retinoic Acid) and Tretinoin (All-Trans Retinoic Acid (ATRA)) Nano Particles as Anti-Cancer Nano Drugs under Synchrotron9 Radiations Using Hartree-Fock (HF) and Density Functional Theory (DFT) Method. Insights in Biomed. 2016;1(2):8. DOI: $10.21767 / 2572-5610.100008$

55. Alireza Heidari. Advances in Logic, Operations and Computational Mathematics. J Appl Computat Math. 2016; 5(5). DOI: 10.4172/21689679.1000e144

56. Alireza Heidari. Mathematical Equations in Predicting Physical Behavior. J Appl Computat Math. 2016;5(5) 2016, DOI: 10.4172/2168-9679.1000e145

57. Alireza Heidari. Chemotherapy a Last Resort for Cancer Treatment. Chemo Open Access. 2016;5(4). DOI: 10.4172/2167-7700.100e130

58. Alireza Heidari. Separation and Pre-Concentration of Metal CationsDNA/RNA Chelates Using Molecular Beam Mass Spectrometry with Tunable Vacuum Ultraviolet (VUV) Synchrotron Radiation and Various Analytical Methods. Mass Spectrom Purif Tech. 2016;2(2). DOI: $10.4172 / 2469-9861.1000 \mathrm{e} 101$

59. Alireza Heidari. Yoctosecond Quantitative Structure-Activity Relationship (QSAR) and Quantitative Structure-Property Relationship (QSPR) under Synchrotron Radiations Studies for Prediction of Solubility of Anti- Cancer Nano Drugs in Aqueous Solutions Using Genetic Function Approximation (GFA) Algorithm. Insight Pharm Res. 2016;1(1).

60. Alireza Heidari. Cancer Risk Prediction and Assessment in Human Cells under Synchrotron Radiations Using Quantitative Structure Activity Relationship (QSAR) and Quantitative Structure Properties Relationship (QSPR) Studies. Int J Clin Med Imaging . 2016;3(10): 516. DOI: $10.4172 / 2376-0249.1000516$

61. Alireza Heidari. A Novel Approach to Biology. Electronic J Biol. 2016;12(4):1-2.

62. Alireza Heidari. Innovative Biomedical Equipment's for Diagnosis and Treatment. J Bioengineer \& Biomedical Sci. 2016;6(2). DOI: 10.4172/2155-9538.1000e124

63. Alireza Heidari. Integrating Precision Cancer Medicine into Healthcare, Medicare Reimbursement Changes and the Practice of 
Oncology: Trends in Oncology Medicine and Practices. J Oncol Med \& Pract. 2016;1(2).

64. Alireza Heidari. Promoting Convergence in Biomedical and Biomaterials Sciences and Silk Proteins for Biomedical and Biomaterials Applications: An Introduction to Materials in Medicine and Bioengineering Perspectives. J Bioengineer \& Biomedical Sci. 2016; 6(3). DOI: 10.4172/2155-9538.1000e126

65. Alireza Heidari. X-Ray Fluorescence and X-Ray Diffraction Analysis on Discrete Element Modeling of Nano Powder Metallurgy Processes in Optimal Container Design. J Powder Metall Min. 2017;6(1). DOI: 10.4172/2168-9806.1000e136

66. Alireza Heidari. Biomolecular Spectroscopy and Dynamics of NanoSized Molecules and Clusters as Cross- Linking-Induced AntiCancer and Immune-Oncology Nano Drugs Delivery in DNA/RNA of Human Cancer Cells' Membranes under Synchrotron Radiations: A Payload-Based Perspective. Arch Chem Res. 2017;1(2):11. DOI: 10.21767/2572-4657.100011

67. Alireza Heidari. Deficiencies in Repair of Double-Standard DNA/ RNA-Binding Molecules Identified in Many Types of Solid and Liquid Tumors Oncology in Human Body for Advancing Cancer Immunotherapy Using Computer Simulations and Data Analysis. J Appl Bioinforma Comput Biol. 2017;6(1). DOI: 10.4172/23299533.1000e 104

68. Alireza Heidari. Electronic Coupling among the Five Nanomolecules Shuts Down Quantum Tunneling in the Presence and Absence of an Applied Magnetic Field for Indication of the Dimer or other Provide Different Influences on the Magnetic Behavior of Single Molecular Magnets (SMMs) as Qubits for Quantum Computing. Glob J Res Rev. 2017;4(2):19. DOI: 10.21767/2393-8854.100019

69. Alireza Heidari. Polymorphism in Nano-Sized Graphene LigandInduced Transformation of Au38- xAgx/xCux(SPh-tBu)24 to Au36$\mathrm{xAgx} / \mathrm{xCux}(\mathrm{SPh}-\mathrm{tBu}) 24(\mathrm{x}=1-12)$ Nanomolecules for Synthesis of Au144- xAgx/xCux[(SR)60, (SC4)60, (SC6)60, (SC12)60, (PET)60, (p-MBA)60, (F)60, (Cl)60, (Br)60, (I)60, (At)60, (Uus)60 and (SC6H13)60] Nano Clusters as Anti-Cancer Nano Drugs. J Nanomater Mol Nanotechnol. 2017; 6(3). DOI: 10.4172/2324-8777.1000e109

70. Alireza Heidari. Biomedical Resource Oncology and Data Mining to Enable Resource Discovery in Medical, Medicinal, Clinical, Pharmaceutical, Chemical and Translational Research and Their Applications in Cancer Research. Int J Biomed Data Min. 2017;6(2). DOI: $10.4172 / 2090-4924.1000 \mathrm{e} 103$

71. Alireza Heidari. Study of Synthesis, Pharmacokinetics, Pharmacodynamics, Dosing, Stability, Safety and Efficacy of Olympiadane Nano molecules as Agent for Cancer Enzymotherapy, Immunotherapy, Chemotherapy, Radiotherapy, Hormone Therapy and Targeted Therapy under Synchrotorn Radiation. J Dev Drugs. 2017;6(2). DOI: 10.4172/2329-6631.1000e15

72. Alireza Heidari. A Novel Approach to Future Horizon of Top Seven Biomedical Research Topics to Watch in 2017: Alzheimer's, Ebola, Hypersomnia, Human Immunodeficiency Virus (HIV), Tuberculosis (TB), Microbiome/Antibiotic Resistance and Endovascular Stroke. J Bioengineer \& Biomedical Sci. 2017;7(1). DOI: $10.4172 / 2155-9538.1000 \mathrm{e} 127$

73. Alireza Heidari. Opinion on Computational Fluid Dynamics (CFD) Technique. Fluid Mech Open Acc. 2017;4(3):157. DOI:10.4172/2476-

\subsection{7}

74. Alireza Heidari.ConcurrentDiagnosis ofOncology Influence Outcomes in Emergency General Surgery for Colorectal Cancer and Multiple Sclerosis (MS) Treatment Using Magnetic Resonance Imaging (MRI) and Au329(SR)84, Au329-xAgx(SR)84, Au144(SR)60, Au68(SR)36, Au30(SR)18, Au102(SPh)44, Au38(SPh)24, Au38(SC2H4Ph)24, Au21S(SAdm)15, Au36(pMBA)24 and Au25(pMBA)18 Nano Clusters. J Surgery Emerg Med. 2017;1(21).

75. Alireza Heidari. Developmental Cell Biology in Adult Stem Cells Death and Autophagy to Trigger a Preventive Allergic Reaction to Common Airborne Allergens under Synchrotron Radiation Using Nanotechnology for Therapeutic Goals in Particular Allergy Shots (Immunotherapy). Cell Biol (Henderson, NV). 2017; 6(1). doi: 10.4172/2324-9293.1000e117

76. Alireza Heidari. Changing Metal Powder Characteristics for Elimination of the Heavy Metals Toxicity and Diseases in Disruption of Extracellular Matrix (ECM) Proteins Adjustment in Cancer Metastases Induced by Osteosarcoma, Chondrosarcoma, Carcinoid, Carcinoma, Ewing's Sarcoma, Fibrosarcoma and Secondary Hematopoietic Solid or Soft Tissue Tumors. J Powder Metall Min. 2017;6(2). DOI: 10.4172/2168-9806.1000170

77. Alireza Heidari. Nanomedicine-Based Combination Anti-Cancer Therapy between Nucleic Acids and Anti- Cancer Nano Drugs in Covalent Nano Drugs Delivery Systems for Selective Imaging and Treatment of Human Brain Tumors Using Hyaluronic Acid, Alguronic Acid and Sodium Hyaluronate as Anti-Cancer Nano Drugs and Nucleic Acids Delivery under Synchrotron Radiation. Am J Drug Deliv. 2017;5(2):092-096. DOI: 10.21767/2321-547X.1000016

78. Alireza Heidari. Clinical Trials of Dendritic Cell Therapies for Cancer Exposing Vulnerabilities in Human Cancer Cells' Metabolism and Metabolomics: New Discoveries, Unique Features Inform New Therapeutic Opportunities, Biotech's Bumpy Road to the Market and Elucidating the Biochemical Programs that Support Cancer Initiation and Progression. J Biol Med Science. 2017;1(1):e103.

79. Alireza Heidari. The Design Graphene-Based Nanosheets as a New Nanomaterial in Anti-Cancer Therapy and Delivery of Chemotherapeutics and Biological Nano Drugs for Liposomal Anti-Cancer Nano Drugs and Gene Delivery. Br Biomed Bull. 2017;5(2):305. DOI: 10.21767/2471-9897.1000305

80. Alireza Heidari. Integrative Approach to Biological Networks for Emerging Roles of Proteomics, Genomics and Transcriptomics in the Discovery and Validation of Human Colorectal Cancer Biomarkers from DNA/RNA Sequencing Data under Synchrotron Radiation. Transcriptomics. 2017; 5(2). DOI: 10.4172/2329-8936.1000e117

81. Alireza Heidari. Elimination of the Heavy Metals Toxicity and Diseases in Disruption of Extracellular Matrix (ECM) Proteins and Cell Adhesion Intelligent Nanomolecules Adjustment in Cancer Metastases Using Metalloenzymes and under Synchrotron Radiation. Lett Health Biol Sci. 2017;2(2):1-4. DOI. 10.15436/24756245.17.019

82. Alireza Heidari. Treatment of Breast Cancer Brain Metastases through a Targeted Nanomolecule Drug Delivery System Based on Dopamine Functionalized Multi-Wall Carbon Nanotubes (MWCNTs) Coated with Nano Graphene Oxide (GO) and Protonated Polyaniline (PANI) in Situ During the Polymerization of Aniline Autogenic 
Nanoparticles for the Delivery of Anti-Cancer Nano Drugs under Synchrotron Radiation. Br J Res. 2017;4(3):16.

83. Alireza Heidari. Sedative, Analgesic and Ultrasound-Mediated Gastrointestinal Nano Drugs Delivery for Gastrointestinal Endoscopic Procedure, Nano Drug-Induced Gastrointestinal Disorders and Nano Drug Treatment of Gastric Acidity. Res Rep Gastroenterol. 2017;1:1.

84. Alireza Heidari. Synthesis, Pharmacokinetics, Pharmacodynamics, Dosing, Stability, Safety and Efficacy of Orphan Nano Drugs to Treat High Cholesterol and Related Conditions and to Prevent Cardiovascular Disease under Synchrotron Radiation. J Pharm Sci Emerg Drugs. 2017;5:1. DOI: 10.4172/2380-9477.1000e104

85. Alireza Heidari. Non-Linear Compact Proton Synchrotrons to Improve Human Cancer Cells and Tissues Treatments and Diagnostics through Particle Therapy Accelerators with Monochromatic Microbeams. J Cell Biol Mol Sci. 2017;2(1):1-5.

86. Alireza Heidari. Design of Targeted Metal Chelation Therapeutics Nanocapsules as Colloidal Carriers and Blood-Brain Barrier (BBB) Translocation to Targeted Deliver Anti-Cancer Nano Drugs into the Human Brain to Treat Alzheimer's Disease under Synchrotron Radiation. J Nanotechnol Material Sci. 2017;4(2):1-5.

87. Ricardo Gobato. Alireza Heidari. Calculations Using Quantum Chemistry for Inorganic Molecule Simulation BeLi2SeSi. American Journal of Quantum Chemistry and Molecular Spectroscopy. 2017;2(3):37-46. Doi: 10.11648/j.ajqcms.20170203.12

88. Alireza Heidari. Different High-Resolution Simulations of Medical, Medicinal, Clinical, Pharmaceutical and Therapeutics Oncology of Human Lung Cancer Translational Anti-Cancer Nano Drugs Delivery Treatment Process under Synchrotron and X-Ray Radiations. J Med Oncol. 2017;1(1):1-2.

89. Alireza Heidari. A Modern Ethnomedicinal Technique for Transformation, Prevention and Treatment of Human Malignant Gliomas Tumors into Human Benign Gliomas Tumors under Synchrotron Radiation. Am J Ethnomed. 2010;4(1):1-4.

90. Alireza Heidari. An Investigation of the Role of DNA as Molecular Computers: A Computational Study on the Hamiltonian Path Problem. International Journal of Scientific \& Engineering Research. 2014;5(1):1884-1889.

91. Alireza Heidari. Active Targeted Nanoparticles for Anti-Cancer Nano Drugs Delivery across the Blood- Brain Barrier for Human Brain Cancer Treatment, Multiple Sclerosis (MS) and Alzheimer's Diseases Using Chemical Modifications of Anti-Cancer Nano Drugs or DrugNanoparticles through Zika Virus (ZIKV) Nanocarriers under Synchrotron Radiation. J Med Chem Toxicol. 2017;2(3):1-5.

92. Alireza Heidari. Investigation of Medical, Medicinal, Clinical and Pharmaceutical Applications of Estradiol, Mestranol (Norlutin), Norethindrone (NET), Norethisterone Acetate (NETA), Norethisterone Enanthate (NETE) and Testosterone Nanoparticles as Biological Imaging, Cell Labeling, Anti-Microbial Agents and AntiCancer Nano Drugs in Nanomedicines Based Drug Delivery Systems for Anti-Cancer Targeting and Treatment. Parana Journal of Science and Education (PJSE). 2017;3(4):10-19.

93. Alireza Heidari. A Comparative Computational and Experimental Study on Different Vibrational Biospectroscopy Methods, Techniques and Applications for Human Cancer Cells in Tumor Tissues Simulation, Modeling, Research, Diagnosis and Treatment.
Open J Anal Bioanal Chem. 2017;1(1):014-020.

94. Alireza Heidari. Combination of DNA/RNA Ligands and Linear/ Non-Linear Visible-Synchrotron Radiation- Driven N-Doped Ordered Mesoporous Cadmium Oxide (CdO) Nanoparticles Photocatalysts Channels Resulted in an Interesting Synergistic Effect Enhancing Catalytic Anti-Cancer Activity. Enz Eng. 2017;6:1. DOI: 10.4172/2329-6674.1000160

95. Alireza Heidari. Modern Approaches in Designing Ferritin, Ferritin Light Chain, Transferrin, Beta-2 Transferrin and BacterioferritinBased Anti-Cancer Nano Drugs Encapsulating Nanosphere as DNABinding Proteins from Starved Cells (DPS). Mod Appro Drug Des. 2017;1(1):1-5.

96. Alireza Heidari. Potency of Human Interferon $\beta-1$ a and Human Interferon $\beta-1 \mathrm{~b}$ in Enzymotherapy, Immunotherapy, Chemotherapy, Radiotherapy, Hormone Therapy and Targeted Therapy of Encephalomyelitis Disseminate/Multiple Sclerosis (MS) and Hepatitis A, B, C, D, E, F and G Virus Enter and Targets Liver Cells. J Proteomics Enzymol. 2017;6:1. Doi: 10.4172/2470-1289.1000e109

97. Alireza Heidari. Transport Therapeutic Active Targeting of Human Brain Tumors Enable Anti-Cancer Nanodrugs Delivery across the Blood-Brain Barrier (BBB) to Treat Brain Diseases Using Nanoparticles and Nanocarriers under Synchrotron Radiation. J Pharm Pharmaceutics. 2017;4(2):1-5.

98. Alireza Heidari, Christopher Brown. Combinatorial Therapeutic Approaches to DNA/RNA and Benzylpenicillin (Penicillin G), Fluoxetine Hydrochloride (Prozac and Sarafem), Propofol (Diprivan), Acetylsalicylic Acid (ASA) (Aspirin), Naproxen Sodium (Aleve and Naprosyn) and Dextromethamphetamine Nanocapsules with Surface Conjugated DNA/RNA to Targeted Nano Drugs for Enhanced Anti-Cancer Efficacy and Targeted Cancer Therapy Using Nano Drugs Delivery Systems. Ann Adv Chem. 2017;1: 061-069.

99. Alireza Heidari. Vibrational Spectroscopy of Nucleic Acids", Wahid Ali Khan (Editor), "Basic Biochemistry", Austin Publishing Group (APG)/Austin Publications LLC, ISBN: 978-0-9971499-2-0, Pages 1-18, Jersey City, New Jersey, USA, 2016.

100. Alireza Heidari. High-Resolution Simulations of Human Brain Cancer Translational Nano Drugs Delivery Treatment Process under Synchrotron Radiation. J Transl Res. 2017;1(1):1-3

101. Alireza Heidari. Investigation of Anti-Cancer Nano Drugs' Effects' Trend on Human Pancreas Cancer Cells and Tissues Prevention, Diagnosis and Treatment Process under Synchrotron and X-Ray Radiations with the Passage of Time Using Mathematica. Current Trends Anal Bioanal Chem. 2017;1(1):36-41.

102. Alireza Heidari. Pros and Cons Controversy on Molecular Imaging and Dynamics of Double-Standard DNA/RNA of Human Preserving Stem Cells-Binding Nano Molecules with Androgens/Anabolic Steroids (AAS) or Testosterone Derivatives through Tracking of Helium-4 Nucleus (Alpha Particle) Using Synchrotron Radiation. Arch Biotechnol Biomed. 2017;1(1):067-0100.

103. Alireza Heidari. Visualizing Metabolic Changes in Probing Human Cancer Cells and Tissues Metabolism Using Vivo 1H or Proton NMR, 13C NMR, 15N NMR and 31P NMR Spectroscopy and Self-Organizing Maps under Synchrotron Radiation. SOJ Mater Sci Eng. 2017;5(2):16.

104. Alireza Heidari. Cavity Ring-Down Spectroscopy (CRDS), Circular 
Dichroism Spectroscopy, Cold Vapour Atomic Fluorescence Spectroscopy and Correlation Spectroscopy Comparative Study on Malignant and Benign Human Cancer Cells and Tissues with the Passage of Time under Synchrotron Radiation. Enliven: Challenges Cancer Detect Ther. 2017;4(2):e001.

105. Alireza Heidari. Laser Spectroscopy, Laser-Induced Breakdown Spectroscopy and Laser-Induced Plasma Spectroscopy Comparative Study on Malignant and Benign Human Cancer Cells and Tissues with the Passage of Time under Synchrotron Radiation. Int J Hepatol Gastroenterol, 2017;3(4):079-084.

106. Alireza Heidari. Time-Resolved Spectroscopy and Time-Stretch Spectroscopy Comparative Study on Malignant and Benign Human Cancer Cells and Tissues with the Passage of Time under Synchrotron Radiation. Enliven: Pharmacovigilance and Drug Safety. 2017;4 (2):e001.

107. Alireza Heidari. Overview of the Role of Vitamins in Reducing Negative Effect of Decapeptyl (Triptorelin Acetate or Pamoate Salts) on Prostate Cancer Cells and Tissues in Prostate Cancer Treatment Process through Transformation of Malignant Prostate Tumors into Benign Prostate Tumors under Synchrotron Radiation. Open J Anal Bioanal Chem. 2017;1(1):021-026.

108. Alireza Heidari. Electron Phenomenological Spectroscopy, Electron Paramagnetic Resonance (EPR) Spectroscopy and Electron Spin Resonance (ESR) Spectroscopy Comparative Study on Malignant and Benign Human Cancer Cells and Tissues with the Passage of Time under Synchrotron Radiation. Austin J Anal Pharm Chem. 2017;4(3):1091.

109. Alireza Heidari. Therapeutic Nanomedicine Different HighResolution Experimental Images and Computational Simulations for Human Brain Cancer Cells and Tissues Using Nanocarriers Deliver DNA/RNA to Brain Tumors under Synchrotron Radiation with the Passage of Time Using Mathematica and MATLAB. Madridge J Nano Tech. Sci. 2017;2(2):77-83.

110. Alireza Heidari. A Consensus and Prospective Study on Restoring Cadmium Oxide (CdO) Nanoparticles Sensitivity in Recurrent Ovarian Cancer by Extending the Cadmium Oxide (CdO) Nanoparticles-Free Interval Using Synchrotron Radiation Therapy as Antibody-Drug Conjugate for the Treatment of Limited-Stage Small Cell Diverse Epithelial Cancers. Cancer Clin Res Rep. 2017;1(2);e001.

111. Alireza Heidari. A Novel and Modern Experimental Imaging and Spectroscopy Comparative Study on Malignant and Benign Human Cancer Cells and Tissues with the Passage of Time under White Synchrotron Radiation.Cancer Sci Res Open Access. 2017;4(2):1-8.

112. Alireza Heidari. Different High-Resolution Simulations of Medical, Medicinal, Clinical, Pharmaceutical and Therapeutics Oncology of Human Breast Cancer Translational Nano Drugs Delivery Treatment Process under Synchrotron and X-Ray Radiations. J Med Oncol. 2017;1(1):12-17.

113. Alireza Heidari. Vibrational Decihertz (dHz), Centihertz (cHz), Millihertz (mHz), Microhertz $(\mu H z)$, Nanohertz $(\mathrm{nHz})$, Picohertz $(\mathrm{pHz})$, Femtohertz $(\mathrm{fHz})$, Attohertz $(\mathrm{aHz})$, Zeptohertz $(\mathrm{zHz})$ and Yoctohertz (yHz) Imaging and Spectroscopy Comparative Study on Malignant and Benign Human Cancer Cells and Tissues under Synchrotron Radiation. International Journal of Biomedicine.
2017;7(4):335-340.

114. Alireza Heidari. Force Spectroscopy and Fluorescence Spectroscopy Comparative Study on Malignant and Benign Human Cancer Cells and Tissues with the Passage of Time under Synchrotron Radiation. EC Cancer. 2017;2 (5):239-246.

115. Alireza Heidari. Photoacoustic Spectroscopy, Photoemission Spectroscopy and Photothermal Spectroscopy Comparative Study on Malignant and Benign Human Cancer Cells and Tissues with the Passage of Time under Synchrotron Radiation. BAOJ Cancer Res Ther. 2017;3(3):45-52.

116. Alireza Heidari. J-Spectroscopy, Exchange Spectroscopy (EXSY), Nucle 7 ar Overhauser Effect Spectroscopy (NOESY) and Total Correlation Spectroscopy (TOCSY) Comparative Study on Malignant and Benign Human Cancer Cells and Tissues under Synchrotron Radiation. EMS Eng Sci J. 2017;1(2): 6-13.

117. Alireza Heidari. Neutron Spin Echo Spectroscopy and Spin Noise Spectroscopy Comparative Study on Malignant and Benign Human Cancer Cells and Tissues with the Passage of Time under Synchrotron Radiation. Int J Biopharm Sci. 2017;1:103-107.

118. Alireza Heidari. Vibrational Decahertz (daHz), Hectohertz (hHz), Kilohertz (kHz), Megahertz (MHz), Gigahertz (GHz), Terahertz (THz), Petahertz (PHz), Exahertz (EHz), Zettahertz (ZHz) and Yottahertz (YHz) Imaging and Spectroscopy Comparative Study on Malignant and Benign Human Cancer Cells and Tissues under Synchrotron Radiation. Madridge J Anal Sci Instrum. 2017;2(1):41-46.

119. Alireza Heidari. Two-Dimensional Infrared Correlation Spectroscopy, Linear Two-Dimensional Infrared Spectroscopy and Non-Linear Two-Dimensional Infrared Spectroscopy Comparative Study on Malignant and Benign Human Cancer Cells and Tissues under Synchrotron Radiation with the Passage of Time. J Mater Sci Nanotechnol. 2018;6(1):101.

120. Alireza Heidari. Fourier Transform Infrared (FTIR) Spectroscopy, Near-Infrared Spectroscopy (NIRS) and Mid-Infrared Spectroscopy (MIRS) Comparative Study on Malignant and Benign Human Cancer Cells and Tissues under Synchrotron Radiation with the Passage of Time. Int J Nanotechnol Nanomed. 2018;3(1):1-6.

121. Alireza Heidari. Infrared Photo Dissociation Spectroscopy and Infrared Correlation Table Spectroscopy Comparative Study on Malignant and Benign Human Cancer Cells and Tissues under Synchrotron Radiation with the Passage of Time. Austin Pharmacol Pharm. 2018;3(1):1011,

122. Alireza Heidari. Novel and Transcendental Prevention, Diagnosis and Treatment Strategies for Investigation of Interaction among Human Blood Cancer Cells, Tissues, Tumors and Metastases with Synchrotron Radiation under Anti-Cancer Nano Drugs Delivery Efficacy Using MATLAB Modeling and Simulation. Madridge J Nov Drug Res. 2017;1 (1):18-24. doi: 10.18689/mjndr.2017-104.

123. Alireza Heidari. Comparative Study on Malignant and Benign Human Cancer Cells and Tissues with the Passage of Time under Synchrotron Radiation. Open Access J Trans Med Res. 2018;2 (1):2632.doi: 10.15406/oajtmr.2018.02.00026

124. Marcia Regina Risso Gobato, Ricardo Gobato, Alireza Heidari. Planting of Jaboticaba Trees for Landscape Repair of Degraded Area. Landscape Architecture and Regional Planning. 2018;3(1):1-9. doi: 


\subsection{8/j.larp.20180301.11}

125. Alireza Heidari. Fluorescence Spectroscopy, Phosphorescence Spectroscopy and Luminescence Spectroscopy Comparative Study on Malignant and Benign Human Cancer Cells and Tissues under Synchrotron Radiation with the Passage of Time. SM J Clin. Med. Imaging. 2018;4 (1):1018.

126. Alireza Heidari. Nuclear Inelastic Scattering Spectroscopy (NISS) and Nuclear Inelastic Absorption Spectroscopy (NIAS) Comparative Study on Malignant and Benign Human Cancer Cells and Tissues under Synchrotron Radiation.Int J Pharm Sci. 2018;2(1):1-14.

127. Alireza Heidari. X-Ray Diffraction (XRD), Powder X-Ray Diffraction (PXRD) and Energy-Dispersive X-Ray Diffraction (EDXRD) Comparative Study on Malignant and Benign Human Cancer Cells and Tissues under Synchrotron Radiation. J Oncol Res. 2018;2(1):114.

128. Alireza Heidari. Correlation Two-Dimensional Nuclear Magnetic Resonance (NMR) (2D-NMR) (COSY) Imaging and Spectroscopy Comparative Study on Malignant and Benign Human Cancer Cells and Tissues under Synchrotron Radiation. EMS Can Sci. 2018;1:1.

129. Alireza Heidari.Thermal Spectroscopy, Photothermal Spectroscopy, Thermal Microspectroscopy, Photothermal Microspectroscopy, Thermal Macrospectroscopy and Photothermal Macrospectroscopy Comparative Study on Malignant and Benign Human Cancer Cells and Tissues with the Passage of Time under Synchrotron Radiation. SM J Biometrics Biostat. 2018;3(1):1024.

130. Alireza Heidari. A Modern and Comprehensive Experimental Biospectroscopic Comparative Study on Human Common Cancers' Cells, Tissues and Tumors before and after Synchrotron Radiation Therapy. Open Acc J Oncol Med. 2018;1(1).

131. Alireza Heidari. Heteronuclear Correlation Experiments such as Heteronuclear Single-Quantum Correlation Spectroscopy (HSQC), Heteronuclear Multiple-Quantum Correlation Spectroscopy (HMQC) and Heteronuclear Multiple-Bond Correlation Spectroscopy (HMBC) Comparative Study on Malignant and Benign Human Endocrinology and Thyroid Cancer Cells and Tissues under Synchrotron Radiation. J Endocrinol Thyroid Res. 2018;3 (1): 555-603. doi: 10.19080/ JETR.2018.03.555604

132. Alireza Heidari. Nuclear Resonance Vibrational Spectroscopy (NRVS), Nuclear Inelastic Scattering Spectroscopy (NISS), Nuclear Inelastic Absorption Spectroscopy (NIAS) and Nuclear Resonant Inelastic X-Ray Scattering Spectroscopy (NRIXSS) Comparative Study on Malignant and Benign Human Cancer Cells and Tissues under Synchrotron Radiation. Int J Bioorg Chem Mol Biol. 2018;6 (1e): $1-5$.

133. Alireza Heidari. A Novel and Modern Experimental Approach to Vibrational Circular Dichroism Spectroscopy and Video Spectroscopy Comparative Study on Malignant and Benign Human Cancer Cells and Tissues with the Passage of Time under White and Monochromatic Synchrotron Radiation. Glob J Endocrinol Metab. 2018;1(3). GJEM. 000514-000519.

134. Alireza Heidari. Pros and Cons Controversy on Heteronuclear Correlation Experiments such as Heteronuclear Single-Quantum Correlation Spectroscopy (HSQC), Heteronuclear Multiple-Quantum Correlation Spectroscopy (HMQC) and Heteronuclear Multiple-Bond Correlation Spectroscopy (HMBC) Comparative Study on Malignant and Benign Human Cancer Cells and Tissues under Synchrotron Radiation. EMS Pharma J. 2018;1(1):002-008.

135. Alireza Heidari. A Modern Comparative and Comprehensive Experimental Biospectroscopic Study on Different Types of Infrared Spectroscopy of Malignant and Benign Human Cancer Cells and Tissues with the Passage of Time under Synchrotron Radiation. J Analyt Molecul Tech. 2018;3(1):8.

136. Alireza Heidari.Investigation of Cancer Types Using Synchrotron Technology for Proton Beam Therapy: An Experimental Biospectroscopic Comparative Study. European Modern Studies Journal. 2018;2(1):13-29.

137. Alireza Heidari. Saturated Spectroscopy and Unsaturated Spectroscopy Comparative Study on Malignant and Benign Human Cancer Cells and Tissues with the Passage of Time under Synchrotron Radiation. Imaging J Clin Medical Sci. 2018;5 (1):001-007.

138. Alireza Heidari. Small-Angle Neutron Scattering (SANS) and WideAngle X-Ray Diffraction (WAXD) Comparative Study on Malignant and Benign Human Cancer Cells and Tissues under Synchrotron Radiation. Int J Bioorg Chem Mol Biol. 2018;6(2e):1-6.

139. Alireza Heidari. Investigation of Bladder Cancer, Breast Cancer, Colorectal Cancer, Endometrial Cancer, Kidney Cancer, Leukemia, Liver, Lung Cancer, Melanoma, Non-Hodgkin Lymphoma, Pancreatic Cancer, Prostate Cancer, Thyroid Cancer and Non-Melanoma Skin Cancer Using Synchrotron Technology for Proton Beam Therapy: An Experimental Biospectroscopic Comparative Study. Ther Res Skin Dis. 2018;1(1).

140. Alireza Heidari. Attenuated Total Reflectance Fourier Transform Infrared (ATR-FTIR) Spectroscopy, Micro-Attenuated Total Reflectance Fourier Transform Infrared (Micro-ATR-FTIR) Spectroscopy and Macro-Attenuated Total Reflectance Fourier Transform Infrared (Macro-ATR-FTIR) Spectroscopy Comparative Study on Malignant and Benign Human Cancer Cells and Tissues under Synchrotron Radiation with the Passage of Time. International Journal of Chemistry Papers. 2018;2(1):1-12.

141. Alireza Heidari. Mössbauer Spectroscopy, Mössbauer Emission Spectroscopy and 57Fe Mössbauer Spectroscopy Comparative Study on Malignant and Benign Human Cancer Cells and Tissues under Synchrotron Radiation. Acta Scientific Cancer Biology. 2018;2(3):1720.

142. Alireza Heidari.Comparative Study on Malignant and Benign Human Cancer Cells and Tissues under Synchrotron Radiation with the Passage of Time. Organic \& Medicinal Chem IJ. 2018;6 (1): 555-676.

143. Alireza Heidari. Correlation Spectroscopy, Exclusive Correlation Spectroscopy and Total Correlation Spectroscopy Comparative Study on Malignant and Benign Human AIDS-Related Cancers Cells and Tissues with the Passage of Time under Synchrotron Radiation. Int J Bioanal Biomed. 2018;2 (1):001-007.

144. Alireza Heidari. Biomedical Instrumentation and Applications of Biospectroscopic Methods and Techniques in Malignant and Benign Human Cancer Cells and Tissues Studies under Synchrotron Radiation and Anti-Cancer Nano Drugs Delivery. Am J Nanotechnol Nanomed. 2018;1(1):001-009.

145. Alireza Heidari. Vivo 1H or Proton NMR, 13C NMR, 15N NMR and 31P NMR Spectroscopy Comparative Study on Malignant and Benign Human Cancer Cells and Tissues under Synchrotron Radiation. Ann Biomet Biostat. 2018;1(1):1001. 


\section{Human Cancer Cells and Tissues with the Passage of Time under Synchrotron Radiation}

146. Alireza Heidari. Grazing-Incidence Small-Angle Neutron Scattering (GISANS) and Grazing-Incidence X-Ray Diffraction (GIXD) Comparative Study on Malignant and Benign Human Cancer Cells, Tissues and Tumors under Synchrotron Radiation. Ann Cardiovasc Surg. 2018;1(2):1006.

147. Alireza Heidari. Adsorption Isotherms and Kinetics of Multi-Walled Carbon Nanotubes (MWCNTs), Boron Nitride Nanotubes (BNNTs), Amorphous Boron Nitride Nanotubes (a-BNNTs) and Hexagonal Boron Nitride Nanotubes (h-BNNTs) for Eliminating Carcinoma, Sarcoma, Lymphoma, Leukemia, Germ Cell Tumor and Blastoma Cancer Cells and Tissues. Clin Med Rev Case Rep.2018;5(1):201.

148. Alireza Heidari.Correlation Spectroscopy (COSY), Exclusive Correlation Spectroscopy (ECOSY), Total Correlation Spectroscopy (TOCSY), Incredible Natural-Abundance Double-Quantum Transfer Experiment (INADEQUATE), Heteronuclear Single-Quantum
Correlation Spectroscopy (HSQC), Heteronuclear Multiple-Bond Correlation Spectroscopy (HMBC), Nuclear Overhauser Effect Spectroscopy (NOESY) and Rotating Frame Nuclear Overhauser Effect Spectroscopy (ROESY) Comparative Study on Malignant and Benign Human Cancer Cells and Tissues under Synchrotron Radiation. Acta Scientific Pharmaceutical Sciences. 2018;2(5):30-35.

149. Alireza Heidari. Pump-Probe Spectroscopy and Transient Grating Spectroscopy Comparative Study on Malignant and Benign Human Cancer Cells and Tissues with the Passage of Time under Synchrotron Radiation. Adv Material Sci Engg. 2018;2(3):1-7.

150. Alireza Heidari. Grazing-Incidence Small-Angle X-Ray Scattering (GISAXS) and Grazing-Incidence Wide-Angle X-Ray Scattering (GIWAXS) Comparative Study on Malignant and Benign Human Cancer Cells and Tissues under Synchrotron Radiation. Insights Pharmacol Pharm Sci. 2018;1(1):1-8. 